\title{
Utilização de água residuária de origem doméstica na agricultura: Estudo das alterações químicas do solo ${ }^{1}$
}

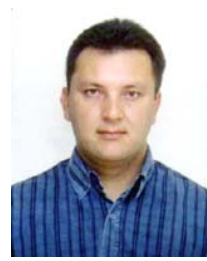

Salomão de S. Medeiros ${ }^{2}$, Antônio A. Soares², Paulo A. Ferreira², Júlio C. L. Neves ${ }^{3}$, Antônio T. de Matos ${ }^{2} \&$ José A. A. de Souza ${ }^{4}$

\footnotetext{
1 Parte da Tese de Doutorado do primeiro autor, apresentada a Universidade Federal de Viçosa - UFV

2 DEA/UFV, CEP 36571-000, Viçosa, MG. E-mail: salommao@gmail.com; aasoares@ufv.br; pafonso@ufv.br; atmatos@ufv.br

3 DPS/UFV, CEP 36571-000, Viçosa, MG

${ }^{4}$ Curso de Agronomia/UFV, CEP 36571-000, Viçosa, MG
}

Protocolo 37 - 5/4/2005 - Aprovado em 31/10/2005

\begin{abstract}
Resumo: Neste trabalho, teve-se como objetivo principal investigar as alterações químicas do solo, em resposta à aplicação de água residuária filtrada de origem doméstica e comparar os resultados com aqueles obtidos com o manejo convencional. $O$ experimento foi implantado na Unidade Piloto de Tratamento de Água Residuária e Agricultura Irrigada, localizada na Universidade Federal de Viçosa - UFV. O delineamento experimental constituiu-se de 18 unidades experimentais, cada uma composta de oito plantas. $O$ experimento foi montado segundo o esquema de parcelas subdivididas, tendo nas parcelas os tipos de manejo adotados (convencional - MC e com água residuária de origem doméstica - MR, com aplicação de cinco diferentes lâminas) e, nas subparcelas as faixas de profundidade do solo $(0-0,20 ; 0,20-0,40$ e 0,40 - 0,60 m) no delineamento em blocos casualizados (linhas de plantio) com três repetições. Os resultados obtidos permitiram concluir que a adoção do MR foi mais efetiva na melhoria das características do solo que o MC.
\end{abstract}

Palavras-chave: esgoto, fertirrigação, café

\section{Application of domestic wastewater in agriculture: Study of the chemical changes in soil}

\begin{abstract}
The objective of this work was to investigate chemical changes in the soil, in response to application of filtered domestic wastewater and to compare the results with the conventional agricultural management. The experiment was carried out at the Pilot Sewer Treatment Plant and Irrigated Agriculture located at Federal University of Viçosa. The experimental design consisted of 18 plots, with eight plants each. The treatments were distributed in split-plots, the main plots being the management types (CM-conventional and WM-domestic wastewater with five different water depths) and the subplots soil depths $(0-0.20 ; 0.20-0.40$ and $0.40-0.60 \mathrm{~m})$ in randomized blocks consisting of three repetitions. According to the results it may be concluded that the application of WM was more effective to improve soil fertility than CM.
\end{abstract}

Key words: wastewater, fertigation, coffee

\section{INTRODUÇÃO}

A água é um recurso natural essencial à vida, ao desenvolvimento econômico e ao bem-estar social. Embora encontrada em abundância no território nacional, já apresenta comprometimento da sua quantidade e qualidade, principalmente nas regiões próximas aos grandes centros. A crescente preocupação com a preservação dos recursos hídricos e a conservação do meio ambiente, têm conduzido à criação de uma legislação mais rigorosa e eficiente, no intuito de proteger a quantidade e qualidade dos recursos ambientais.
Procurando adequar-se a esta nova política, a sociedade vem buscando tecnologias de menor custo que minimizem os efeitos negativos de suas atividades impactantes. Dentre as tecnologias disponíveis para o tratamento de água residuária de origem doméstica ou destino final dos efluentes líquidos, destaca-se o método de disposição de água no solo, cuja técnica vem sendo utilizada em grande escala, em várias locais do mundo, sobretudo em regiões áridas e semi-áridas. No processo de tratamento por disposição no solo utiliza-se do sistema solo-planta para a degradação, assimilação e imobilização dos constituintes da água residuária e dos produtos de sua transformação no meio. 
Atualmente, e devido ao elevado consumo de água pela agricultura e em razão da sua escassez, muitos países têm optado pelo aproveitamento de águas residuárias na agricultura (disposição de água no solo), em particular as de origem urbana (Metcalf \& Eddy, 1991). Segundo van der Hoek et al. (2002), as maiores vantagens do aproveitamento da água residuária, são: conservação da água disponível, sua grande disponibilidade, possibilitar o aporte e a reciclagem de nutrientes (reduzindo a necessidade de fertilizantes químicos) e concorrer para a preservação do meio ambiente.

Os efeitos da aplicação de água residuária nas propriedades químicas do solo, só são pronunciados após longo período de aplicação, pelos parâmetros que definem sua composição física e química, pelas condições de clima e pelo tipo de solo. Ayers \& Westcot (1999) relatam que a limitação principal do uso de águas residuárias na agricultura é a sua composição química (totais de sais dissolvidos, presença de íons tóxicos e concentração relativa de sódio) e a tolerância das culturas a este tipo de efluente. Segundo Pizarro (1990), os sais solúveis contidos nas águas de irrigação podem, em certas condições climáticas, salinizar o solo e modificar a composição iônica no complexo sortivo, alterando as características físicas e químicas do solo, como o regime de umidade, aeração, nutrientes, desenvolvimento vegetativo e produtividade.

Neste sentido objetivou-se, com este trabalho, investigar as alterações químicas do solo, em resposta à aplicação de água residuária filtrada de origem doméstica e comparar os resultados com aqueles obtidos com o manejo convencional.

\section{MATERIAL E MÉTODOS}

O experimento foi realizado na Unidade Piloto de Tratamento de Água Residuária e Agricultura Irrigada, localizada na Universidade Federal de Viçosa - UFV, pertencente ao Departamento de Engenharia Agrícola-DEA. A Unidade Piloto é constituída por uma estação elevatória e uma unidade de tratamento de água residuária de origem doméstica (abastecida pelo esgoto proveniente do condomínio Bosque Acamari) conjugada a um sistema de irrigação localizada por gotejamento, que possibilita a aplicação do efluente em uma área de 0,14 ha de cafeeiro (variedade Catuaí) com três anos de idade, cujo espaçamento é de $2,5 \mathrm{~m}$ entre linhas de plantio e $0,75 \mathrm{~m}$ entre plantas. A classificação do solo da área experimental, segundo Vieira (2003), é um Cambissolo Háplico Tb distrófico latossólico, dividido em cinco horizontes denominados: horizonte $\mathrm{A}$ de $0 \mathrm{a}$ $0,13 \mathrm{~m}$ de profundidade; horizonte $\mathrm{AB}$ de 0,13 a $0,26 \mathrm{~m}$ de profundidade; horizonte $\mathrm{BA}$ de 0,26 a $0,48 \mathrm{~m}$; horizonte $\mathrm{B}_{1}$ de 0,48 a $0,75 \mathrm{~m}$ e o horizonte $\mathrm{B}_{2}$ de 0,75 a $1,00 \mathrm{~m}$.

$\mathrm{Na}$ realização do experimento usou-se apenas parte da Unidade Piloto, na qual se montou uma infra-estrutura para aplicação da água residuária bruta, oriunda do esgoto doméstico (Figura 1). A infra-estrutura é composta de uma linha de derivação que capta a água residuária bruta da adutora e a conduz a um filtro de areia, para ser filtrada; após a filtragem, a água residuária é armazenada em tanque com capacidade de $2.500 \mathrm{~L}$, o qual possui um sistema de motobomba acoplado, possibilitando a sua aplicação através de um sistema de irrigação por gotejamento após filtrada novamente, por um filtro de disco.
A.

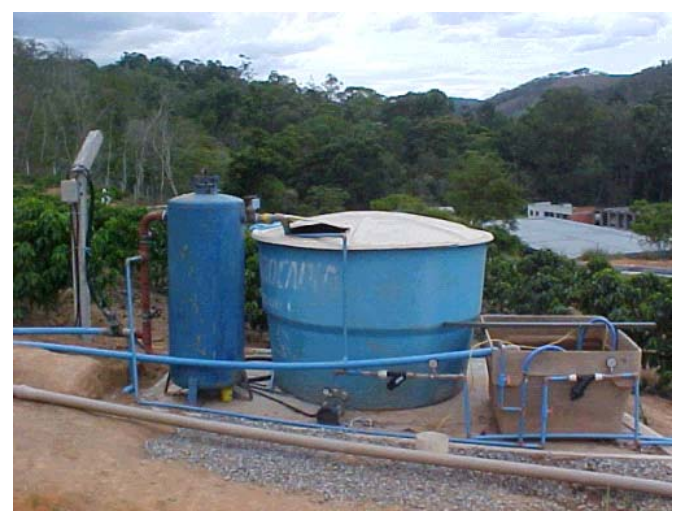

B.

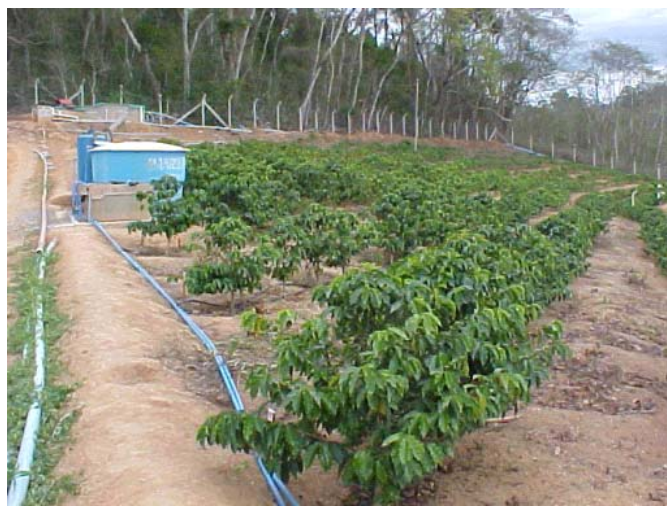

Figura 1. Infra-estrutura (A) montada para aplicação da água residuária filtrada (B)

O filtro de areia tem formato cilíndrico com fluxo ascendente tendo, como material filtrante, camadas sobrepostas de cascalho, areia grossa lavada, areia fina lavada, areia grossa lavada e cascalho, conforme ilustrado na Figura 2A; já o filtro de disco (Figura 2B) é de 1" de 120 mesh, com capacidade de filtragem de até $5,0 \mathrm{~m}^{3} \mathrm{~h}^{-1}$. Por outro lado, o filtro de areia trabalhava com capacidade de filtragem de aproximadamente $0,2 \mathrm{~m}^{3} \mathrm{~h}^{-1}$.

Anterior à implantação desta infra-estrutura, realizaram-se coleta de solo em diversos pontos da área selecionada para determinação de suas características físicas e químicas, que

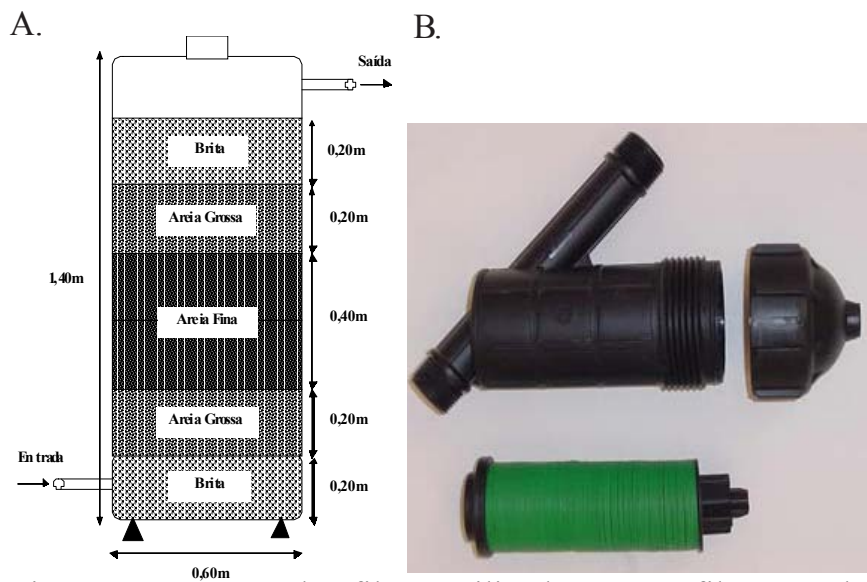

Figura 2. Esquema dos filtros utilizados para a filtragem da água residuária bruta, de origem doméstica: (A) filtro de areia e (B) filtro de disco 
representaram a condição inicial $\left(\mathrm{C}_{0}\right)$, as quais foram realizadas com o auxílio de um trado tipo holandês, nas faixas de profundidade de $0-0,20 ; 0,20-0,40$ e $0,40-0,60 \mathrm{~m}$.

Nos Laboratórios de Física e de Fertilidade do Solo, ambos do Departamento de Solos - DPS da UFV, foram determinadas as características químicas do solo: condutividade elétrica do extrato da pasta saturada do solo (CE), razão de adsorção de sódio (RAS), porcentagem de sódio trocável (PST), $\mathrm{pH}, \mathrm{P}, \mathrm{K}^{+}$, $\mathrm{Na}^{+}, \mathrm{Ca}^{2+}, \mathrm{Mg}^{2+}, \mathrm{Al}^{3+}, \mathrm{H}+\mathrm{Al}$, matéria orgânica (MO), $\mathrm{N}$ - total, $\mathrm{P}$ - remanescente e $\mathrm{S}$, segundo metodologias descritas em EMBRAPA (1997).

O delineamento experimental adotado totalizou 18 unidades experimentais, com oito plantas cada uma, ocupando uma área de $15 \mathrm{~m}^{2}$. O experimento foi montado segundo o esquema de parcelas subdivididas, tendo nas parcelas os tipos de manejo adotados (convencional - MC e com água residuária - MR, com aplicação de cinco diferentes lâminas) e, nas subparcelas, as faixas de profundidade $(0-0,20 ; 0,20-0,40$ e $0,40-0,60 \mathrm{~m})$ no delineamento em blocos casualizados (linhas de plantio) com três repetições.

O período de monitoramento das alterações químicas do solo foi de 270 dias, realizando-se, a cada 90 dias, coleta do solo. O experimento foi iniciado em novembro de 2003 e concluído em agosto de 2004. As amostras de solo foram coletadas paralelamente à linha de plantio, nos dois lados, a uma distância de aproximadamente $0,10 \mathrm{~m}$ do caule do cafeeiro. Estas foram realizadas nas quatro plantas centrais de cada unidade experimental, totalizando oito amostras simples, para formar uma composta.

O MC (tratamento $\mathrm{T}_{1}$ ), consistiu de calagem, adubação convencional e irrigação suplementar com água da represa. Realizaram-se a calagem e a adubação do cafeeiro com base na análise química do solo, seguindo a recomendação da Comissão de Fertilidade do Solo do Estado de Minas Gerais (1999). A calagem foi realizada com base no método da neutralização do $\mathrm{Al}^{3+}$ e da elevação dos teores de $\mathrm{Ca}^{2+} \mathrm{e} \mathrm{Mg}^{2+}$ aplicando-lhe, de uma só vez, $1.000 \mathrm{~kg} \mathrm{ha}^{-1}$ ano $^{-1}$ de calcário em linha contínua, abaixo da saia do cafeeiro. Na adubação convencional foram aplicados $50 \mathrm{~kg} \mathrm{ha}^{-1}$ ano-1 de $\mathrm{P}_{2} \mathrm{O}_{5}$, tomando-se por base as concentrações de $\mathrm{P}$ disponível no solo e a de $\mathrm{P}$ remanescente, considerando-se uma produtividade 31 a $40 \mathrm{sc} \mathrm{ha}^{-1}$. A fonte de $\mathrm{P}_{2} \mathrm{O}_{5}$ utilizada foi o superfosfato simples, aplicado de uma só vez; por outro lado, a adubação com $\mathrm{N} \mathrm{e} \mathrm{K}^{+}$foi realizada através de aplicações de $300 \mathrm{~kg} \mathrm{ha}^{-1} \mathrm{ano}^{-1}$ de $\mathrm{N}$ e de $\mathrm{K}_{2} \mathrm{O}$, com base na concentração de $\mathrm{N}$ nas folhas e na concentração de $\mathrm{K}^{+}$no solo. A aplicação desses nutrientes foi realizada abaixo da saia do cafeeiro e parcelada três vezes, com intervalo de 30 dias. A fonte de $\mathrm{N}$ utilizada foi o sulfato de amônia e a de $\mathrm{K}$, o cloreto de potássio. A irrigação foi realizada no $\mathrm{MC}$, com base na evapotranspiração do cafeeiro, sendo que, no tempo $1-\mathrm{T}_{\mathrm{p} 1}$ (após 90 dias da adoção dos manejos) aplicou-se uma lâmina acumulada de $46 \mathrm{~mm}$; no tempo $2-\mathrm{T}_{\mathrm{p} 2}$ (após 180 dias da adoção dos manejos) a lâmina acumulada totalizou $55 \mathrm{~mm}$ e, no tempo $3-\mathrm{T}_{\mathrm{p} 3}$ (após 270 dias da adoção dos manejos) a lâmina acumulada foi de $101 \mathrm{~mm}$.

Para estimativa da evapotranspiração do cafeeiro instalouse uma estação climatológica na área experimental, para monitoramento das condições climáticas.
No MR, o critério adotado na definição das lâminas de água residuária de origem doméstica baseou-se no aporte de $\mathrm{N}$ total ao solo; as lâminas aplicadas teriam de aportar o equivalente a 200, 300, 400, 500 e $600 \mathrm{~kg} \mathrm{ha}^{-1} \mathrm{ano}^{-1} \mathrm{de} \mathrm{N}$ - total ao solo, aos tratamentos $\mathrm{T}_{2}, \mathrm{~T}_{3}, \mathrm{~T}_{4}, \mathrm{~T}_{5}$ e $\mathrm{T}_{6}$, respectivamente; contudo, as lâminas a serem aplicadas totalizariam 401, 600, 802 , 1001 e $1201 \mathrm{~mm}^{2} \mathrm{ano}^{-1}$, considerando-se uma concentração média de $\mathrm{N}$ - total na água residuária de $50 \mathrm{mg} \mathrm{L}^{-1}$; todavia, em razão das precipitações ocorridas no período as lâminas de água residuária aplicadas acumuladas, totalizaram: 117, 146, 234, 264 e 293 mm no T $_{\mathrm{pl}}$, no $\mathrm{T}_{\mathrm{p} 2} 155,197,309,360$ e $399 \mathrm{~mm}$ e no $\mathrm{T}_{\mathrm{p} 3} 202,262,399,468$ e $532 \mathrm{~mm}$.

A freqüência da aplicação da água residuária foi diária, exceto aos sábados e domingos; a cada aplicação era coletada uma alíquota da água residuária no ponto de aplicação (gotejador), posteriormente armazenada no freezer; ao final de cada mês e com as alíquotas coletadas, fazia-se uma amostra composta e se determinava o $\mathrm{pH}, \mathrm{CE}$ e as concentrações de $\mathrm{P}$, $\mathrm{K}^{+}, \mathrm{N}$ - total, $\mathrm{Na}^{+}, \mathrm{Ca}^{2+}, \mathrm{Mg}^{2+}, \mathrm{Zn}, \mathrm{Cu}, \mathrm{Fe}$ e $\mathrm{Mn}$, segundo metodologia proposta pela APHA (1995 e 1998). Com este monitoramento, foi possível estimar-se quantitativamente o aporte desses nutrientes ao solo.

Os dados foram analisados para cada tempo $\left(\mathrm{T}_{\mathrm{p} 1}, \mathrm{~T}_{\mathrm{p} 2}, \mathrm{e} \mathrm{T}_{\mathrm{p} 3}\right)$ nas faixas de profundidade de $0-0,20 ; 0,20-0,40$ e $0,40-0,60$ $\mathrm{m}$, em função do tipo de manejo adotado (MC e MR); de início, realizou-se a análise de variância para cada atributo monitorado em cada tempo.

Com base no modelo estatístico, realizou-se o desdobramento da interação tratamento x faixa de profundidade, no sentido de se estudar os efeitos das diferentes lâminas de água residuária aplicadas $\left(\mathrm{T}_{2}, \mathrm{~T}_{3}, \mathrm{~T}_{4}, \mathrm{~T}_{5}\right.$ e $\left.\mathrm{T}_{6}\right)$ em cada faixa de profundidade; contudo, ajustaram-se equações de regressão aos dados coletados, em função da lâmina aplicada em cada faixa de profundidade e em cada tempo.

Em virtude de não se observar, na grande maioria dos casos (87\%), diferenças estatísticas significativas dos atributos do solo em função das lâminas de água residuária aplicada, optouse por realizar uma estatística descritiva dos atributos do solo monitorado e testar um contraste (com o nível máximo de significância de $5 \%$ de probabilidade) a fím de se verificar as diferenças médias dos atributos do solo em cada faixa de profundidade e em cada tempo, em resposta ao tipo de manejo adotado (MC e MR).

Para as análises estatísticas, utilizou-se o software SAEG 5.0(FUNARBE, 1993).

\section{RESULTADOS E DISCUSSÃO}

\section{Caracterização da água residuária e aporte de nutrientes ao solo}

As Tabelas 1 e 2 apresentam as características químicas e físicas, respectivamente, da água residuária filtrada de origem doméstica utilizada no experimento. Dentre os atributos monitorados, os que apresentaram menor coeficiente de variação foram o pH e CE (5,68 e 8,09\%, respectivamente); os demais mostraram grande variação, sobretudo o $\mathrm{P}, \mathrm{N}$ - total e $\mathrm{Cu}$.

Tendo em vista as diretrizes apresentadas por Ayers \& Westcot (1999) vê-se que, em se tratando de qualidade de água para irrigação quanto a salinidade, a água residuária filtrada de 
Tabela 1. Características químicas da água residuária filtrada de origem doméstica utilizada no experimento

\begin{tabular}{|c|c|c|c|c|c|c|c|c|c|c|c|c|c|}
\hline \multirow{2}{*}{ Mês } & \multirow{2}{*}{ pH } & $\mathrm{CEa}$ & $\mathrm{P}$ & K & N-Total & $\mathrm{Na}$ & $\mathrm{Ca}$ & $\mathrm{Mg}$ & $\mathrm{Zn}$ & $\mathrm{Cu}$ & $\mathrm{Fe}$ & $\mathrm{Mn}$ & \multirow{2}{*}{$\begin{array}{c}\text { RAS } \\
\left(\mathrm{mmol}_{\mathrm{c}} \mathrm{L}^{-1}\right)^{0,5}\end{array}$} \\
\hline & & $\left(\mathrm{dS} \mathrm{m}^{-1}\right)$ & \multicolumn{10}{|c|}{$\mathrm{mg} \mathrm{L}^{-1}$} & \\
\hline Nov & 6,84 & 0,57 & 7,02 & 37,79 & 25,04 & 56,31 & 14,06 & 2,82 & 0,21 & 2,08 & 11,19 & 0,22 & 3,58 \\
\hline Dez & 7,39 & 0,58 & 6,26 & 37,79 & 23,79 & 55,42 & 9,33 & 2,63 & 0,08 & 0,49 & 2,99 & 0,08 & 4,13 \\
\hline Jan & 7,61 & 0,58 & 6,83 & 28,15 & 27,68 & 45,52 & 8,17 & 2,73 & 0,16 & 0,23 & 10,76 & 0,25 & 3,52 \\
\hline Fev & 6,41 & 0,58 & 55,21 & 29,02 & 178,33 & 38,33 & 8,28 & 2,08 & 0,05 & 0,05 & 2,77 & 0,04 & 3,08 \\
\hline Mar & 7,06 & 0,55 & 7,07 & 22,17 & 37,56 & 41,22 & 5,44 & 0,69 & 0,02 & 0,26 & 5,00 & 0,04 & 4,42 \\
\hline Abr & 7,17 & 0,49 & 10,42 & 52,24 & 33,40 & 72,55 & 11,83 & 4,28 & 0,02 & 0,09 & 1,67 & 0,00 & 4,59 \\
\hline Mai & 7,68 & 0,47 & 5,66 & 17,59 & 31,16 & 20,32 & 19,88 & 3,84 & 0,05 & 0,20 & 6,48 & 0,18 & 1,09 \\
\hline Jun & 7,51 & 0,59 & 7,23 & 29,89 & 26,71 & 26,74 & 22,64 & 4,42 & 0,08 & 0,05 & 2,17 & 0,12 & 1,35 \\
\hline Jul & 7,44 & 0,60 & 8,02 & 36,04 & 48,41 & 32,25 & 25,24 & 5,52 & 0,02 & 0,05 & 3,61 & 0,12 & 1,51 \\
\hline Média & 7,23 & 0,56 & 12,64 & 32,30 & 48,01 & 43,18 & 13,87 & 3,22 & 0,08 & 0,39 & 5,18 & 0,12 & 3,03 \\
\hline CV (\%) & 5,68 & 8,09 & 126,80 & 31,44 & 103,03 & 37,78 & 51,09 & 44,81 & 88,83 & 167,21 & 69,38 & 72,83 & 45,21 \\
\hline IC & 0,27 & 0,03 & 10,47 & 6,63 & 32,31 & 10,66 & 4,63 & 0,94 & 0,04 & 0,42 & 2,35 & 0,06 & 0,90 \\
\hline
\end{tabular}

CEa: Condutividade elétrica da água; $\mathrm{CV}$ : Coeficiente de variação e IC: Intervalo de confiança

Tabela 2. Características físicas da água residuária filtrada de origem doméstica, utilizada no experimento

\begin{tabular}{lc}
\hline Atributos & ${ }^{1}$ Valor $\left(\mathrm{mg} \mathrm{L}^{-1}\right)$ \\
\hline Sólidos sedimentáveis ${ }^{2}$ & 1,00 \\
Sólidos totais & 528,00 \\
Sólidos fixos & 245,00 \\
Sólidos voláteis & 283,00 \\
Sólidos suspensos totais & 34,00 \\
Sólidos dissolvidos totais & 494,00 \\
TMédia de três determinações & \\
${ }^{2}$ A unidade de sólidos sedimentáveis está em $\mathrm{mL} \mathrm{L}^{-1}$ &
\end{tabular}

origem doméstica não apresentou qualquer grau de restrição de uso durante o período de monitoramento, por apresentar uma $\mathrm{CEa}<0,7 \mathrm{dS} \mathrm{m}^{-1}$; todavia, avaliando-se quanto ao aspecto problemas de infiltração, a água residuária apresentou grau de restrição de ligeiro a moderado. No que se refere à toxicidade de íons específicos, caso do $\mathrm{Na}^{+}$, a água residuária filtrada não indicou restrições de uso, em razão de apresentar concentração menor que $69 \mathrm{mg} \mathrm{L}^{-1}$, durante o período monitorado.

$\mathrm{O}$ valor médio do $\mathrm{pH}$ da água residuária, segundo classificação de Feigin et al. (1991) é considerado médio, portanto, com a faixa normal para uso na irrigação; já as concentrações médias de $\mathrm{P}, \mathrm{K}, \mathrm{N}$ - total, $\mathrm{Na}, \mathrm{Ca}+\mathrm{Mg}$, são consideradas, segundo os mesmos autores, média-alta, alta, média-alta, média-baixa, baixa, respectivamente, evidenciando o potencial de uso da água residuária como fonte de nutrientes. As concentrações médias de Zn e Mn na água residuária filtrada de origem doméstica, estão de acordo com as diretrizes para uso na irrigação por longo período; contudo, as concentrações médias de $\mathrm{Cu}$ e $\mathrm{Fe}$ estão um pouco acima do recomendado se a lâmina aplicada for maior que $1.200 \mathrm{~mm} \mathrm{ano}^{-1}$.

Quanto à influência da qualidade da água residuária no surgimento de problemas de obstrução no sistema de irrigação localizada, verifica-se que a concentração de sólidos suspensos não apresenta nenhum grau de restrição, segundo as diretrizes estabelecidas por Feigin et al. (1991), mas possui grau de restrição de ligeira a moderada para o pH e Mn; no que se refere à concentração de $\mathrm{Fe}$, a água residuária possui severa restrição, por apresentar concentração maior $1,5 \mathrm{mg} \mathrm{L}^{-1}$.
Com base nas concentrações de nutrientes encontradas na água residuária e nas lâminas aplicadas em cada tempo, estimou-se o potencial fertilizante da água residuária filtrada, proveniente do esgoto doméstico (Tabela 3), porém esta estimativa não contempla um aspecto fundamental, que é a forma como os nutrientes se encontram no efluente.

De acordo com as características iniciais do solo (Tabela 4), as estimativas de aporte de nutrientes ao solo e as exigências nutricionais do cafeeiro, constata-se que a aplicação da água residuária filtrada de origem doméstica ao final do experimento $\left(\mathrm{T}_{\mathrm{p} 3}\right)$, só foi capaz de suprir adequadamente as necessidades de $\mathrm{P}$, com aplicação da maior lâmina $\left(\mathrm{T}_{6}\right)$. Esta lâmina foi capaz de suprir 72 e $58 \%$ da demanda de $\mathrm{N}$ e K, respectivamente. Em relação aos micronutrientes ( $\mathrm{Zn}, \mathrm{Cu}$ e $\mathrm{Mn})$, a aplicação desta lâmina supriria apenas a necessidade de $\mathrm{Cu}$, considerando-se um solo com baixa disponibilidade.

A quantidade de sódio aplicada ao solo ao fim do experimento, variou de 92,93 a 241,9 $\mathrm{kg} \mathrm{ha}^{-1}$. Essas taxas de aplicação de sódio ao solo são bastante elevadas quando comparadas com solos que receberam irrigação com água superficial com baixa concentração de sódio e condutividade elétrica menor que $0,70 \mathrm{dS} \mathrm{m}^{-1}$ podendo, então, constituir fator limitante para o uso da água residuária filtrada de origem doméstica na agricultura, em razão de haver possibilidade de acúmulo de sódio, ao longo do tempo; no entanto, práticas especiais de manejo, como aplicação de lâminas de lixiviação, aplicação de água superficial em determinados períodos e a suspensão da aplicação da água residuária durante o período chuvoso, podem contribuir para a lixiviação do sódio abaixo da zona radicular, evitando problemas de toxicidade.

\section{Características iniciais do solo e durante o período de monitoramento}

Encontram-se, na Tabela 4, os valores médios dos atributos do solo (pH, $\mathrm{P}, \mathrm{K}^{+}, \mathrm{Na}^{+}, \mathrm{Ca}^{2+}, \mathrm{Mg}^{2+}, \mathrm{Al}^{3+}, \mathrm{H}+\mathrm{Al}, \mathrm{MO}, \mathrm{N}-$ total, $\mathrm{P}$ - rem, S, CE, ADA, RAS, e PST) para cada tempo e faixa de profundidade, em função do tipo de manejo adotado (MC e MR).

De acordo com as características químicas do solo no início do experimento e as diretrizes preconizadas pela Comissão de 
Tabela 3. Aporte de nutrientes aplicados ao solo, via água residuária, durante o período de monitoramento

\begin{tabular}{|c|c|c|c|c|c|c|c|c|c|c|c|}
\hline \multirow{2}{*}{ Tratamentos } & \multirow{2}{*}{$\begin{array}{c}\text { Lâminas } \\
\text { Acumuladas (mm) }\end{array}$} & $\mathrm{P}$ & K & N-Total & $\mathrm{Na}$ & $\mathrm{Ca}$ & $\mathrm{Mg}$ & $\mathrm{Zn}$ & $\mathrm{Cu}$ & $\mathrm{Fe}$ & $\mathrm{Mn}$ \\
\hline & & \multicolumn{10}{|c|}{$\left(\mathrm{kg} \mathrm{ha}^{-1}\right)$} \\
\hline & & \multicolumn{10}{|c|}{ Tempo $1\left(T_{p 1}\right)$} \\
\hline $\mathrm{T}_{2}$ & 117 & 7,76 & 41,30 & 29,49 & 62,18 & 12,16 & 3,18 & 0,16 & 1,03 & 8,66 & 0,19 \\
\hline $\mathrm{T}_{3}$ & 146 & 9,74 & 51,46 & 36,85 & 77,54 & 15,55 & 3,97 & 0,21 & 1,42 & 11,48 & 0,25 \\
\hline $\mathrm{T}_{4}$ & 234 & 15,53 & 82,59 & 58,99 & 124,34 & 24,32 & 6,35 & 0,32 & 2,06 & 17,32 & 0,39 \\
\hline $\mathrm{T}_{5}$ & 264 & 17,57 & 93,09 & 66,63 & 140,22 & 27,74 & 7,18 & 0,37 & 2,42 & 20,12 & 0,44 \\
\hline \multirow[t]{2}{*}{$\mathrm{T}_{6}$} & 293 & 19,53 & 103,07 & 73,92 & 155,35 & 31,16 & 7,97 & 0,42 & 2,83 & 23,07 & 0,51 \\
\hline & & \multicolumn{10}{|c|}{ Tempo $2\left(T_{p 2}\right)$} \\
\hline $\mathrm{T}_{2}$ & 155 & 17,63 & 53,79 & 63,26 & 80,61 & 15,29 & 4,01 & 0,17 & 1,08 & 9,88 & 0,20 \\
\hline $\mathrm{T}_{3}$ & 197 & 23,28 & 68,15 & 83,26 & 102,25 & 19,75 & 5,08 & 0,23 & 1,49 & 13,16 & 0,27 \\
\hline $\mathrm{T}_{4}$ & 309 & 35,25 & 107,25 & 126,47 & 160,74 & 30,50 & 7,99 & 0,34 & 2,16 & 19,75 & 0,41 \\
\hline $\mathrm{T}_{5}$ & 360 & 41,56 & 124,20 & 149,43 & 186,55 & 35,52 & 9,22 & 0,40 & 2,56 & 23,30 & 0,47 \\
\hline \multirow[t]{2}{*}{$\mathrm{T}_{6}$} & 399 & 46,86 & 137,97 & 167,66 & 206,99 & 39,89 & 10,28 & 0,45 & 2,97 & 26,52 & 0,54 \\
\hline & & \multicolumn{10}{|c|}{ Tempo $3\left(T_{p 3}\right)$} \\
\hline $\mathrm{T}_{2}$ & 202 & 20,86 & 66,57 & 80,54 & 92,93 & 25,87 & 6,17 & 0,19 & 1,13 & 11,99 & 0,27 \\
\hline $\mathrm{T}_{3}$ & 262 & 27,70 & 85,58 & 106,92 & 119,09 & 34,22 & 8,03 & 0,25 & 1,56 & 16,07 & 0,36 \\
\hline $\mathrm{T}_{4}$ & 399 & 41,41 & 131,60 & 159,44 & 184,21 & 50,65 & 12,11 & 0,38 & 2,26 & 23,77 & 0,54 \\
\hline $\mathrm{T}_{5}$ & 468 & 49,02 & 153,59 & 189,43 & 214,93 & 59,89 & 14,20 & 0,45 & 2,68 & 28,20 & 0,63 \\
\hline $\mathrm{T}_{6}$ & 532 & 56,03 & 174,14 & 216,89 & 241,90 & 69,88 & 16,42 & 0,51 & 3,13 & 32,55 & 0,73 \\
\hline
\end{tabular}

Tabela 4. Alterações químicas do solo em resposta à adoção do tipo de manejo, nos tempos 1, 2 e 3

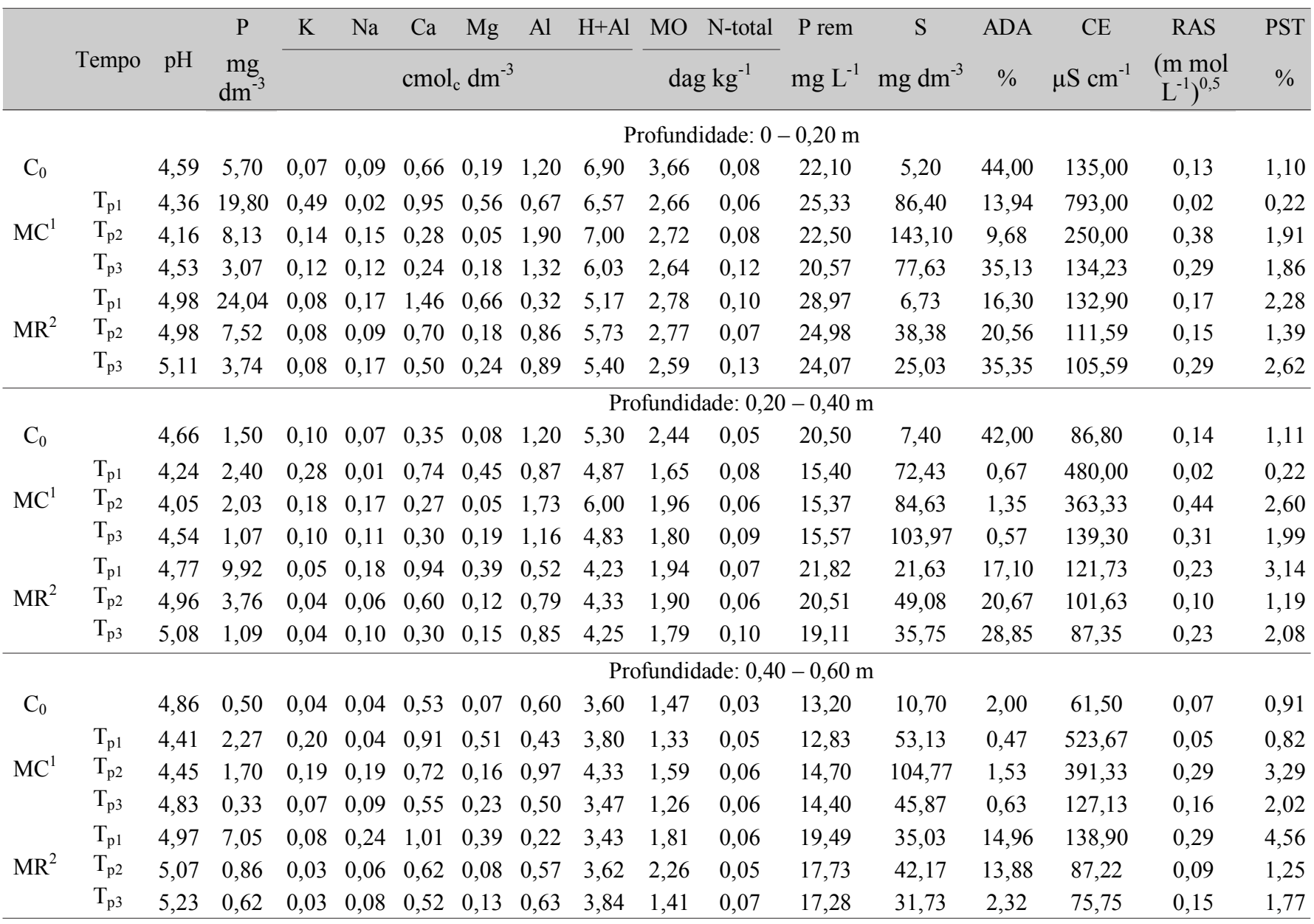

$\mathrm{C}_{0}$ : Condição inicial do solo; $\mathrm{MC}$ : Manejo convencional; $\mathrm{MR}$ : Manejo com água residuária; $\mathrm{T}_{\mathrm{p} 1}, \mathrm{~T}_{\mathrm{p} 2}$ e $\mathrm{T}_{\mathrm{p} 3}$ referente aos tempos 1, 2 e 3, respectivamente

${ }^{1}$ Valores das variáveis no manejo convencional provêm da média das 3 repetições

${ }^{2}$ Valores das variáveis no manejo com água residuária se originam da média geral dos tratamentos $\mathrm{T}_{2}, \mathrm{~T}_{3}, \mathrm{~T}_{4}, \mathrm{~T}_{5}$ e $\mathrm{T}_{6}$ 
Fertilidade do Solo do Estado de Minas Gerais (1999) o solo apresentava acidez elevada ( $\mathrm{pH}$ variando de $4,5-5,0$ ) nas três faixas de profundidade avaliadas, ressaltando-se que o valor de $\mathrm{pH}$ aumentava com a profundidade. A concentração de $\mathrm{P}$ disponível foi considerada muito baixa e as concentrações de

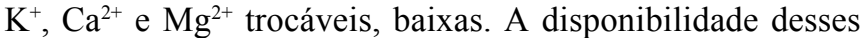
elementos decrescia com a profundidade. A concentração de $\mathrm{S}$ disponível foi considerada baixa na faixa de profundidade de 0 $-0,20 \mathrm{~m}$; média, na de $0,20-0,40 \mathrm{~m}$ e alta na de $0,40-0,60 \mathrm{~m}$. A acidez trocável $\left(\mathrm{Al}^{3+}\right)$ e a potencial $(\mathrm{H}+\mathrm{Al})$ foram consideradas altas nas faixas de profundidade de $0-0,20$ e $0,20-0,40$ e médias na de $0,40-0,60 \mathrm{~m}$.

O conteúdo de matéria orgânica (MO) no solo, nas faixas de profundidade de $0-0,20$ e $0,20-0,40$, foi considerado médio e baixo na de $0,40-0,60 \mathrm{~m}$.

Nas duas primeiras faixas de profundidade, em que o valor da ADA alcançou valores superiores a $40 \%$, foram detectados indícios de que o solo apresentava considerável grau de dispersão.

$\mathrm{Na}$ avaliação conjunta da CE e da PST, notou-se que o solo não apresenta problemas de salinidade e o sódio adsorvido se encontra abaixo dos limites prejudiciais. Segundo Pizarro (1990), este solo é classificado como normal, em virtude de apresentar $\mathrm{CE}<2 \mathrm{dS} \mathrm{m}^{-1}$ e PST $<7 \%$.

De modo geral, observaram-se (Tabela 4), alterações positivas ao longo do tempo em cada faixa de profundidade para a maioria dos atributos avaliados, em resposta à adoção do MC e MR. Nota-se, com a adoção do MC, diminuição do pH do solo, tanto em profundidade quanto no tempo, em relação a $\mathrm{C}_{0}$. Este decréscimo do $\mathrm{pH}$ do solo no $\mathrm{MC}$ foi influenciado pela aplicação de sulfato de amônio $\left(\mathrm{NH}_{4}^{+}\right)$como fonte de nitrogênio que, após o processo de nitrificação, provocou maior acidez do solo, e pela absorção dos cátions básicos $\left(\mathrm{Ca}^{2+}, \mathrm{Mg}^{2+} \mathrm{e} \mathrm{K}^{+}\right)$ da solução do solo pelo cafeeiro. $\mathrm{Na}$ adoção do $\mathrm{MR}$, o pH do solo aumentou, tanto em profundidade como no tempo, o que pode ser atribuído à adição de bases $\left(\mathrm{Ca}^{2+}, \mathrm{Mg}^{2+}, \mathrm{K}^{+}\right.$e $\left.\mathrm{Na}^{+}\right)$ pela água residuária filtrada e por sua característica de alcalinidade ( $\mathrm{pH}$ médio 7,23).

$\mathrm{Na}$ Tabela 5 estão, quantificadas, as diferenças médias (MR - MC) nos atributos monitorados do solo, em cada faixa de profundidade e em cada tempo. De acordo com os dados apresentados, o incremento do $\mathrm{pH}$ do solo no MR foi estatisticamente significativo em relação ao $\mathrm{MC}$, em todas as faixas de profundidade monitoradas. Este aumento ocorreu em todos os tempos $\left(\mathrm{T}_{\mathrm{p} 1}, \mathrm{~T}_{\mathrm{p} 2}\right.$ e $\left.\mathrm{T}_{\mathrm{p} 3}\right)$ embora no $\mathrm{T}_{\mathrm{p} 2}$, o MR tenha possibilitado maior incremento em relação ao MC. Ante o exposto, infere-se que o MR foi mais efetivo no aumento do $\mathrm{pH}$ do solo, comparativamente ao MC, que recebeu calagem. Resultados semelhantes foram obtidos por Johns \& McConchie (1994a); Schipper et al. (1996); Falkiner \& Smith (1997); AlNakshabandi et al.(1997); Speir et al.1(999); Fonseca (2001); Garcia (2003) e Rezende (2003).

No que se refere à concentração de $\mathrm{P}$ disponível no solo, os dois tipos de manejo aumentaram a concentração somente $\operatorname{nos} \mathrm{T}_{\mathrm{p} 1}$ e $\mathrm{T}_{\mathrm{p} 2}$, com relação a $\mathrm{C}_{0}$ (Tabela 5). O aumento da concentração de $\mathrm{P}$ disponível no solo foi mais efetivo na faixa de profundidade de $0-0,20 \mathrm{~m}$, independente do tipo de manejo. De acordo com a Tabela 5, vê-se que o incremento de $\mathrm{P}$ disponível no solo foi maior no MR que no MC (que recebeu aplicação de superfosfato simples), com exceção do $T_{p 2}$ nas faixas de profundidade $0-0,20$ e $0,40-0,60 \mathrm{~m}$; embora não se tenha verificado diferenças significativas, a aplicação de água residuária de origem doméstica ao solo foi tão efetiva como fonte de $\mathrm{P}$ quanto a aplicação de superfosfato simples. Aumentos nos teores de $\mathrm{P}$ em solos irrigados com efluente provenientes de esgoto, têm sido observados por diversos pesquisadores (Latterell et al., 1982; Stewart et al., 1990 e AlNakshabandi et al., 1997) e esses incrementos também são notados, principalmente na camada superficial do solo.

A concentração de $\mathrm{K}^{+}$trocável no solo aumentou em resposta à adoção do $\mathrm{MC}$ em todos os tempos e em todas as profundidades monitoradas em relação a $\mathrm{C}_{0}$, com exceção da faixa de profundidade $0,20-0,40 \mathrm{~m}$, no $\mathrm{T}_{\mathrm{p} z}$; já com a adoção do $\mathrm{MR}$, o aumento se deu somente na faixa de profundidade 0 $0,20 \mathrm{~m}$, e esta referida concentração foi mantida nos tempos avaliados (Tabela 4). $\mathrm{O}$ incremento na concentração de $\mathrm{K}^{+}$ trocável ocorrido foi, no $\mathrm{MC}$ em relação ao $\mathrm{MR}$, significativamente superior, seja em profundidade como no tempo (Tabela 5). Apesar desse incremento, influenciado pela aplicação de cloreto de potássio, ocorreu decréscimo da concentração de $\mathrm{K}$ no solo ao longo do tempo, em resposta à absorção deste elemento pelo cafeeiro. Com o exposto e apesar da água residuária filtrada de origem doméstica possuir alta concentração de $\mathrm{K}$ (média de $32,3 \mathrm{mg} \mathrm{L}^{-1}$ ), as quantidades adicionadas não foram suficientes para elevar sua concentração no solo, devido à grande exigência do cafeeiro. Com relação à concentração de $\mathrm{K}^{+}$trocável no solo, os resultados encontrados na literatura são divergentes. Stewart et al. (1990) observaram que a aplicação de Água Residuária de Origem Doméstica Tratada ocasionou diminuição do teor de $\mathrm{K}^{+}$, enquanto Cromer et al. (1984) e Al-Nakshabandi et al. (1997) verificaram a ocorrência de aumento na concentração de $\mathrm{K}^{+}$ trocável; por outro lado, Feigin et al. (1991) afirmam que, mesmo que haja aumento na concentração de $\mathrm{K}^{+}$disponível mediante a disposição de águas residuárias ao solo, a quantidade desse nutriente exigido pelas plantas é tão elevada que dificilmente apenas a irrigação com efluente poderia suprir adequadamente as plantas.

A adoção do MR aumentou a concentração de $\mathrm{Na}^{+}$trocável no solo, tanto em profundidade como no tempo, com relação a $\mathrm{C}_{0}$ (Tabela 4); entretanto, no $\mathrm{T}_{\mathrm{p} 2}$, em razão das precipitações ocorridas $(540 \mathrm{~mm})$, a concentração diminuiu em relação ao $\mathrm{T}_{\mathrm{pl}}$ $\mathrm{e}$, posteriormente, voltou a crescer no $\mathrm{T}_{\mathrm{p} 3}$. No MC se deu o contrário: no $T_{p 1}$ houve decréscimo da concentração em relação $\mathrm{a}_{0}$, no $\mathrm{T}_{\mathrm{p} 2}$ acréscimo e, no $\mathrm{T}_{\mathrm{p} 3}$ decréscimo. $\mathrm{O}$ acréscimo da concentração de $\mathrm{Na}^{+}$trocável no solo verificado no $T_{p 1}$ em resposta à adoção do $\mathrm{MR}$, foi significativamente superior ao $\mathrm{MC}$; já no $\mathrm{T}_{\mathrm{p} 2} \mathrm{o}$ acréscimo foi significativamente maior no $\mathrm{MC}$ que no $M R$ e, no $T_{p 3}$, o MR foi significativamente maior, mas apenas na primeira profundidade (Tabela 5). $\mathrm{O}$ aumento na concentração de $\mathrm{Na}^{+}$no solo observado no MR, se deve ao fato da água residuária de origem doméstica apresentar concentração média de $43,18 \mathrm{mg} \mathrm{L}^{-1}$; já a diminuição da concentração de $\mathrm{Na}^{+}$trocável do solo no $\mathrm{MC}$, no $\mathrm{T}_{\mathrm{pl}}$, nas camadas de $0-0,20$ e $0,20-0,40 \mathrm{~m}$ em relação a $\mathrm{C}_{0}$, pode ser

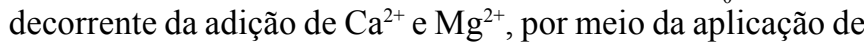
calcário e de superfosfato simples (que possui de 18 a $20 \%$ de 
Tabela 5. Diferenças médias (MR - MC) nos atributos monitorados do solo, em cada profundidade e em cada tempo

\begin{tabular}{|c|c|c|c|c|c|c|c|c|}
\hline \multirow{2}{*}{ Tempo } & \multirow{2}{*}{ Variável } & \multirow{2}{*}{ Unidade } & \multicolumn{6}{|c|}{ Faixa de profundidade (m) } \\
\hline & & & $0-0,20$ & & $0,20-0,40$ & & $0,40-0,60$ & \\
\hline 1 & $\mathrm{pH}$ & & 0,615 & $* *$ & 0,535 & $*$ & 0,560 & $* *$ \\
\hline 2 & & & 0,817 & $* *$ & 0,905 & $* *$ & 0,620 & $* *$ \\
\hline 3 & & & 0,575 & $* *$ & 0,542 & $* *$ & 0,393 & $* *$ \\
\hline 1 & $\mathrm{P}$ & $\mathrm{mg} \mathrm{dm}{ }^{-3}$ & 4,240 & ns & 7,520 & ns & 4,780 & ns \\
\hline 2 & & & $-0,613$ & ns & 1,727 & ns & $-0,840$ & ns \\
\hline 3 & & & 0,673 & $\mathrm{~ns}$ & 0,027 & ns & 0,287 & $\mathrm{~ns}$ \\
\hline 1 & $\mathrm{~K}$ & $\mathrm{cmol}_{\mathrm{c}} \mathrm{dm}^{-3}$ & $-0,416$ & $* *$ & $-0,234$ & $* *$ & $-0,121$ & $* *$ \\
\hline 2 & & & $-0,062$ & $* *$ & $-0,141$ & $* *$ & $-0,161$ & $* *$ \\
\hline 3 & & & $-0,045$ & ** & $-0,060$ & ** & $-0,043$ & ** \\
\hline 1 & $\mathrm{Na}$ & $\mathrm{cmol}_{\mathrm{c}} \mathrm{dm}^{-3}$ & 0,153 & $* *$ & 0,165 & $* *$ & 0,196 & $* *$ \\
\hline 2 & & & $-0,052$ & $* *$ & $-0,111$ & $* *$ & $-0,132$ & $* *$ \\
\hline 3 & & & 0,042 & $* *$ & $-0,012$ & ns & $-0,010$ & ns \\
\hline 1 & $\mathrm{Ca}$ & $\mathrm{cmol}_{\mathrm{c}} \mathrm{dm}^{-3}$ & 0,511 & $*$ & 0,192 & ns & 0,099 & ns \\
\hline 2 & & & 0,422 & ns & 0,333 & ns & $-0,100$ & ns \\
\hline 3 & & & 0,253 & $\mathrm{~ns}$ & 0,007 & ns & $-0,032$ & $\mathrm{~ns}$ \\
\hline 1 & $\mathrm{Mg}$ & $\mathrm{cmol}_{\mathrm{c}} \mathrm{dm}^{-3}$ & 0,103 & $\mathrm{~ns}$ & $-0,064$ & ns & $-0,119$ & ns \\
\hline 2 & & & 0,127 & $\mathrm{~ns}$ & 0,069 & ns & $-0,079$ & ns \\
\hline 3 & & & 0,065 & $\mathrm{~ns}$ & $-0,047$ & ns & $-0,096$ & $* *$ \\
\hline 1 & $\mathrm{Al}$ & cmolc dm-3 & $-0,347$ & $*$ & $-0,347$ & ns & $-0,213$ & $*$ \\
\hline 2 & & & $-1,040$ & $* *$ & $-0,947$ & $* *$ & $-0,400$ & $*$ \\
\hline 3 & & & $-0,426$ & $*$ & $-0,307$ & ns & 0,127 & ns \\
\hline 1 & $\mathrm{H}+\mathrm{Al}$ & $\mathrm{cmol}_{\mathrm{c}} \mathrm{dm}^{-3}$ & $-1,393$ & $* *$ & $-0,633$ & ns & $-0,366$ & ns \\
\hline 2 & & & $-1,273$ & $* *$ & $-1,667$ & $* *$ & $-0,713$ & $*$ \\
\hline 3 & & & $-0,633$ & $* *$ & $-0,580$ & ns & 0,373 & $\mathrm{~ns}$ \\
\hline 1 & MO & dag $\mathrm{kg}^{-1}$ & 0,113 & $\mathrm{~ns}$ & 0,291 & ns & 0,481 & $\mathrm{~ns}$ \\
\hline 2 & & & 0,048 & ns & $-0,063$ & ns & 0,668 & ns \\
\hline 3 & & & $-0,050$ & $\mathrm{~ns}$ & $-0,005$ & ns & 0,151 & $\mathrm{~ns}$ \\
\hline 1 & $\mathrm{~N}-$ total & dag $\mathrm{kg}^{-1}$ & 0,034 & $*$ & $-0,007$ & $\mathrm{~ns}$ & 0,005 & $\mathrm{~ns}$ \\
\hline 2 & & & $-0,008$ & $\mathrm{~ns}$ & $-0,006$ & ns & $-0,013$ & $*$ \\
\hline 3 & & & 0,009 & ns & 0,006 & ns & 0,011 & $\mathrm{~ns}$ \\
\hline 1 & $\mathrm{P}-\mathrm{rem}$ & $m g L^{-1}$ & 3,640 & $\mathrm{~ns}$ & 6,420 & $*$ & 6,660 & $* *$ \\
\hline 2 & & & 2,480 & ns & 5,140 & $* *$ & 3,027 & $\mathrm{~ns}$ \\
\hline 3 & & & 3,507 & $*$ & 3,540 & $*$ & 2,880 & ns \\
\hline 1 & S & $\mathrm{mg} \mathrm{dm} \mathrm{m}^{-3}$ & $-79,673$ & $* *$ & $-50,800$ & $* *$ & $-18,107$ & ns \\
\hline 2 & & & $-104,720$ & $* *$ & $-35,553$ & ns & $-62,600$ & $*$ \\
\hline 3 & & & $-52,600$ & $* *$ & $-68,213$ & $* *$ & $-14,133$ & ns \\
\hline 1 & $\mathrm{ADA}$ & $\%$ & 2,359 & ns & 16,422 & $* *$ & 14,490 & $*$ \\
\hline 2 & & & 10,881 & $* *$ & 19,327 & $* *$ & 12,347 & $\mathrm{~ns}$ \\
\hline 3 & & & 0,217 & ns & 28,287 & $* *$ & 1,683 & ns \\
\hline 1 & $\mathrm{CE}$ & $\mu \mathrm{S} \mathrm{cm}^{-1}$ & $-660,100$ & $* *$ & $-358,267$ & $* *$ & $-384,763$ & $* *$ \\
\hline 2 & & & $-138,413$ & $* *$ & $-261,707$ & $* *$ & $-304,113$ & $* *$ \\
\hline 3 & & & $-28,640$ & $*$ & $-51,949$ & $* *$ & $-51,380$ & * \\
\hline 1 & RAS & $\left(\mathrm{cmol}_{\mathrm{c}} \mathrm{L}^{-1}\right)^{0,5}$ & 0,147 & $* *$ & 0,210 & $* *$ & 0,236 & $* *$ \\
\hline 2 & & & $-0,221$ & $* *$ & $-0,338$ & $* *$ & $-0,196$ & $* *$ \\
\hline 3 & & & 0,001 & ns & $-0,073$ & ns & $-0,008$ & ns \\
\hline 1 & PST & $\%$ & 2,061 & $* *$ & 2,913 & $* *$ & 3,732 & $* *$ \\
\hline 2 & & & $-0,517$ & $\mathrm{~ns}$ & $-1,410$ & $* *$ & $-2,040$ & $* *$ \\
\hline 3 & & & 0,763 & $* *$ & 0,092 & ns & $-0,250$ & ns \\
\hline
\end{tabular}

$\mathrm{Ca}^{2+}$ ), que deslocou o $\mathrm{Na}^{+}$do complexo de troca, seja, por sua valência ou pela alta concentração desses íons no solo. Outro fator que pode ter contribuído para a diminuição do $\mathrm{Na}^{+}$nas duas primeiras faixas de profundidade, refere-se às chuvas e 
às irrigações realizadas com água da represa, que podem ter lixiviado o $\mathrm{Na}^{+}$para as camadas mais inferiores. Vários são os relatos na literatura referentes ao aumento da concentração de $\mathrm{Na}^{+}$trocável no solo, devido à aplicação de águas residuárias (Cromer et al., 1984; Smith et al., 1996; Falkiner \& Smith, 1997; Fonseca, 2001 e Garcia, 2003) e o aumento é influenciado pela concentração na água.

Notou-se que a concentração de $\mathrm{Ca}^{2+}$ trocável no solo aumentou em resposta à adoção do $\mathrm{MR}$, nos $\mathrm{T}_{\mathrm{p} 1}$ e $\mathrm{T}_{\mathrm{p} 2}$, com relação a $\mathrm{C}_{0}$ (Tabela 4), mas se observou, também, que a concentração de $\mathrm{Ca}^{2+}$ decresceu ao longo do tempo, enquanto no $\mathrm{MC}$ este aumento se deu apenas no $\mathrm{T}_{\mathrm{pl}}$; posteriormente, decresceu com relação a $\mathrm{C}_{0}$, com exceção da profundidade 0,40 $-0,60 \mathrm{~m}$. De acordo com os dados apresentados na Tabela 5, o acréscimo da concentração de $\mathrm{Ca}^{2+}$ trocável no solo foi superior no MR que no MC, que recebeu calagem e adubação com superfosfato simples. Constatou-se, ainda, que este incremento só foi significativo no $\mathrm{T}_{\mathrm{pl}}$, na profundidade $0-0,20 \mathrm{~m}$, evidenciando que a aplicação de água residuária foi mais efetiva como fonte de $\mathrm{Ca}^{2+}$ que a aplicação de calcário e adubação com superfosfato simples.

$\mathrm{O}$ aumento da concentração de $\mathrm{Mg}^{2+}$ trocável no solo se deu em resposta à adoção dos dois tipos de manejo em relação a $\mathrm{C}_{0}$ (Tabela 4); no entanto, no MR ocorreu aumento no $\mathrm{T}_{\mathrm{p} 1}$ com relação a $\mathrm{C}_{0}$; posteriormente, decresceu no $\mathrm{T}_{\mathrm{p} 2}$, em relação ao $\mathrm{T}_{\mathrm{p} 1}$, e voltou a aumentar no $\mathrm{T}_{\mathrm{p} 3}$. No $\mathrm{MC}$, o aumento foi somente no $\mathrm{T}_{\mathrm{p} 1}$ em relação a $\mathrm{C}_{0}$. $\mathrm{No}_{\mathrm{p} 2}$, houve decréscimo até em relação a $\mathrm{C}_{0}$ nas duas primeiras camadas e depois retornou a aumentar no $\mathrm{T}_{\mathrm{p} 3}$, em relação ao $\mathrm{T}_{\mathrm{p} 2}$. Não foram constatadas diferenças signifícativas no incremento das concentrações de $\mathrm{Mg}^{2+}$ trocável no solo, em resposta ao tipo de manejo adotado (Tabela 5), evidenciando que a aplicação da água residuária filtrada de origem doméstica, pode ser uma alternativa de aporte de $\mathrm{Mg}^{2+}$ ao solo, em substituição às fontes tradicionais (calagem). Vários registros na literatura especializada relatam aumento (Johns \& McConchie, 1994b; Falkiner \& Smith, 1997) nas concentrações de $\mathrm{Ca}^{2+}$ e $\mathrm{Mg}^{2+}$ trocável no solo, em resposta à aplicação de águas residuárias; no entanto, o acréscimo ou decréscimo deste elemento está diretamente relacionado à concentração na água residuária aplicada, à concentração absorvida pelas plantas e à lixiviação no perfil do solo.

De modo geral, a acidez trocável $\left(\mathrm{Al}^{3+}\right)$ do solo diminuiu em resposta à adoção dos dois tipos de manejo em relação a $\mathrm{C}_{0}$ (Tabela 4). No MR, a diminuição da acidez trocável em relação $\mathrm{a} \mathrm{C}_{0}$ se deu no tempo e em profundidade, com exceção do $\mathrm{T}_{\mathrm{p} 3}$, na faixa de profundidade $0,40-0,60 \mathrm{~m}$. Verificou-se que no $T_{p 1}$ houve a maior redução da acidez trocável no solo; posteriormente, a acidez trocável voltou a crescer em relação ao $\mathrm{T}_{\mathrm{pl}}$ mas, apesar do aumento da acidez, esta ficou abaixo do nível da $\mathrm{C}_{0}$. No $\mathrm{MC}$, a diminuição da acidez trocável em relação a $\mathrm{C}_{0}$ só ocorreu no $\mathrm{T}_{\mathrm{p} 1}$, com exceção do $\mathrm{T}_{\mathrm{p} 3}$, nas faixas de profundidade $0,20-0,40$ e $0,40-0,60 \mathrm{~m}$. De acordo com a Tabela 5, o MR foi superior ao MC (que recebeu calagem) na diminuição da acidez trocável do solo, cuja diminuição foi significativa no tempo e em profundidade, com exceção dos $\mathrm{T}_{\mathrm{p} 1}$ (na faixa de profundidade de $0,20-0,40 \mathrm{~m}$ ) e $\mathrm{T}_{\mathrm{p} 3}$ (nas faixas de profundidade de $0,20-0,40$ e $0,40-0,60 \mathrm{~m}$ ); contudo, conclui- se que o MR foi mais efetivo na diminuição da acidez trocável do solo que o MC, fato que pode ser atribuído ao aumento da alcalinidade do solo e dos cátions trocáveis $\left(\mathrm{Ca}^{2+}, \mathrm{Mg}^{2+} \mathrm{e} \mathrm{Na}^{+}\right)$ influenciada pela aplicação da água residuária.

De modo semelhante à acidez trocável, a potencial $(\mathrm{H}+\mathrm{Al})$ diminuiu em resposta à adoção dos dois tipos de manejo, em relação a $\mathrm{C}_{0}$ (Tabela 4). Verificou-se, no $\mathrm{T}_{\mathrm{pl}}$ diminuição da acidez potencial do solo em ambos os manejos, enquanto no $T_{p 2}$ ocorreu acréscimo em relação ao $T_{p 1}$ no $M R$ e, no $M C$, este aumento foi superior ao valor obtido na $\mathrm{C}_{0}$; por outro lado, este aumento da acidez potencial no MC pode ter sido influenciado pela aplicação de sulfato de amônio $\left(\mathrm{NH}_{4}^{+}\right)$que, durante o processo de nitrificação, aumentou a concentração de $\mathrm{H}^{+}$. Durante o $\mathrm{T}_{\mathrm{p} 3}$, a acidez potencial do solo, em ambos os manejos, voltou a decrescer em relação ao $\mathrm{T}_{\mathrm{p} 2}$, cuja redução foi devida ao aumento da alcalinidade do solo e dos cátions trocáveis $\left(\mathrm{Ca}^{2+}, \mathrm{Mg}^{2+} \mathrm{e} \mathrm{Na}{ }^{+}\right)$; no entanto, no que se refere à diminuição da acidez potencial do solo o MR foi significativamente superior ao MC, sobretudo na camada de 0 $-0,20 \mathrm{~m}$ (Tabela 5). Diminuição da acidez trocável e potencial têm sido verificadas em solos que recebem aplicação de águas residuárias (Johns \& McConchie, 1994b e Falkiner \& Smith, 1997) e esta diminuição é atribuída ao aumento dos cátions trocáveis no solo $\left(\mathrm{Ca}^{2+}, \mathrm{Mg}^{2}, \mathrm{~K}^{+}\right.$e $\left.\mathrm{Na}^{+}\right)$e da alcalinidade do efluente aplicado.

Em geral, a concentração de MO no solo decresceu em relação $\mathrm{a}_{0}$, em virtude da adoção dos dois tipos de manejo (Tabela 4). Este decréscimo foi mais evidente nas primeiras duas camadas de profundidade, mas o decréscimo da concentração de $\mathrm{MO}$ no $\mathrm{MR}$ foi menor que no $\mathrm{MC}$, na maioria dos casos, embora não se tenha verificado efeito significativo (Tabela 5). O maior decréscimo na concentração da MO no solo em resposta ao MC, pode ser atribuído ao efeito da calagem e aplicação de sulfato de amônio, o que favoreceu a atividade microbiana do solo, provocando aceleração da decomposição da MO.

No MR, a concentração de $\mathrm{N}$ - total aumentou em todas as camadas de profundidade e em todos os tempos, com exceção do $\mathrm{T}_{\mathrm{p} 2}$, na faixa de profundidade $0-0,20 \mathrm{~m}$, na qual ocorreu diminuição na concentração desse nutriente, em relação a $\mathrm{C}_{0}$; já no $\mathrm{MC}$, o decréscimo na concentração de $\mathrm{N}$ - total se deu somente nas primeiras faixas de profundidade $(0-0,20 \mathrm{~m})$, nos $\mathrm{T}_{\mathrm{p} 1}$ e $\mathrm{T}_{\mathrm{p} 2}$, em relação a $\mathrm{C}_{0}$ (Tabela 4). De acordo com a Tabela 5 não se verificaram, de forma geral, diferenças significativas no incremento da concentração de $\mathrm{N}$ - total no solo, em função do tipo de manejo adotado, com o que se pressupõe que: se a adoção do MR não incrementar a concentração de $\mathrm{N}$ - total no solo, também não provocará diminuição em relação ao MC.

As concentrações de $\mathrm{S}$ disponíveis no solo aumentaram em relação a $\mathrm{C}_{0}$, em resposta à adoção dos dois tipos de manejo (Tabela 4); todavia, verificou-se que o incremento da concentração de $\mathrm{S}$ disponível no solo foi mais efetivo com a adoção do MC que no MR (Tabela 5). Tal incremento foi estatisticamente significativo, com exceção dos $\mathrm{T}_{\mathrm{p} 1}$ e $\mathrm{T}_{\mathrm{p} 3}$ (na profundidade de $0,40-0,60 \mathrm{~m}$ ) e no $\mathrm{T}_{\mathrm{p} 2}$ (na profundidade de $0,20-0,40 \mathrm{~m})$.

No que se refere à porcentagem de $\mathrm{ADA}$, foi notória a diminuição em relação a $\mathrm{C}_{0}$ em resposta à adoção do $\mathrm{MC}$, em todas as profundidades e em todos os tempos monitorados. 
No MR, a diminuição da porcentagem de ADA ocorreu em todos os tempos, mas só nas faixas de profundidade $0-0,20 \mathrm{e}$ $0,20-0,40 \mathrm{~m}$ (Tabela 4). De acordo com a Tabela 5 a diminuição da porcentagem ADA foi mais pronunciada no MC que no MR, e esta redução foi influenciada principalmente pelo aumento da condutividade elétrica do extrato da pasta saturada do solo (CE) e pela diminuição na concentração de $\mathrm{Na}^{+}$.

Em geral, a $\mathrm{CE}$ do solo aumentou em relação a $\mathrm{C}_{0}$, em resposta ao tipo de manejo adotado, porém no MR se verificou, na profundidade de $0-0,20 \mathrm{~m}$, diminuição em relação a $\mathrm{C}_{0}$; já no $\mathrm{MC}$ a diminuição só ocorreu na $\mathrm{T}_{\mathrm{p} 3}$, na faixas de profundidade de $0-0,20 \mathrm{~m}$ (Tabela 4). Notou-se que o MC foi mais efetivo no incremento da $\mathrm{CE}$ do solo que no MR; contudo, tal incremento foi significativo no tempo e em profundidade (Tabela 5); referido incremento da $\mathrm{CE}$ do solo no $\mathrm{MC}$, é resultado da adubação realizada principalmente pela aplicação de sulfato de amônio. Apesar do aumento da $\mathrm{CE}$ do solo em ambos os manejos, não foram observaram problemas de salinização, por apresentarem $\mathrm{CE}<2000 \mu \mathrm{Scm}^{-1}$.

Constatam-se, nos dados apresentados na Tabela 4, que com a adoção do MC ocorreu redução da RAS do solo em relação a $\mathrm{C}_{0}$ apenas no $\mathrm{T}_{\mathrm{p} 1}$. Com a adoção do $\mathrm{MR}$, esta redução só foi verificada no $T_{p 2}$, na profundidade de $0,20-0,40 \mathrm{~m}$. Apesar do aumento generalizado da RAS do solo nos dois manejos, notou-se que no $\mathrm{T}_{\mathrm{p} 1}$ (profundidade de $0-0,20 ; 0,20-$ 0,40 e $0,40-0,60 \mathrm{~m}$ ) e $\mathrm{T}_{\mathrm{p} 3}$ (profundidade de $0-0,20 \mathrm{~m}$ ) a adoção do MR foi significativamente mais efetiva no aumento da RAS do solo que no MC (Tabela 5). O aumento da RAS do solo, verificado em ambos os manejos, foi atribuído ao aumento da

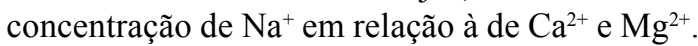

A PST do solo diminuiu apenas no $\mathrm{T}_{\mathrm{p} 1}$ em relação a $\mathrm{C}_{0}$, em resposta à adoção do $\mathrm{MC}$ (Tabela 4). Embora se tenha verificado aumento da PST do solo em resposta à adoção dos manejos, não se constataram, porém, danos à estrutura do solo (PST $<7$ $\%$ ). De acordo com a Tabela 5 , nos $\mathrm{T}_{\mathrm{pl}}$ (profundidade de $0-$ 0,$20 ; 0,20-0,40$ e $0,40-0,60 \mathrm{~m}$ ) e $\mathrm{T}_{\mathrm{p} 3}$ (profundidade de $0-0,20$ e $0,20-0,40 \mathrm{~m}$ ) a adoção do MR foi significativamente mais efetiva no aumento da PST do solo que no MC.

Avaliando-se, em conjunto, a CE e PST do solo em ambos os manejos, vê-se que o solo não apresenta problemas de salinidade nem de danos à sua estrutura, sendo classificado como solo normal, por apresentar uma $\mathrm{CE}<2000 \mu \mathrm{S} \mathrm{cm}^{-1}$ e PST $<7 \%$ (Pizarro, 1990).

\section{CONCLUSÕES}

1. A implantação do MR foi mais efetivo na melhoria da fertilidade do solo que o MC.

2. Os principais impactos positivos observados no solo em resposta à adoção do $\mathrm{MR}$, foram: aumento do $\mathrm{pH}$, das concentrações de $\mathrm{P}$ e $\mathrm{S}$ disponível, $\mathrm{K}^{+}, \mathrm{Ca}^{2+}, \mathrm{Mg}^{2+}$ trocáveis, $\mathrm{MO}, \mathrm{N}$-total e diminuição da acidez trocável e potencial e argila dispersa na água.

3. Os impactos negativos verificados no solo em decorrência do MR, foram: incremento nas concentrações de $\mathrm{Na}^{+}$trocável, aumento da CE, RAS e PST. Apesar do aumento da CE e da PST do solo no MR, não se constataram problemas de salinidade no solo.
4. Do ponto de vista ambiental, a disposição de água residuária no solo pode vir como alternativa para o tratamento dessas águas, além de potencializar a produção de alimentos.

\section{LITERATURA CITADA}

Al-Nakshabandi, G.A.; Saqqar, M.M.; Shatanawi, M.R.; Fayyad, M.; Al-Horani, H. Some environmental problems associated with the use of treated wastewater for irrigation in Jordan. Agricultural Water Management, Amsterdam, v.34, n.1, p.8194, 1997.

APHA - American Public Health Association. Standard methods for the examination of water and wastewater. 19.ed. Washington: APHA, AWWA, WPCR, 1995. 1134p

APHA - American Public Health Association. Standard Methods for the examination of Water and Wastewater. 20.ed. New York: APHA, AWWA, WPCR, 1998. 1325p

Ayers, R.S.; Westcot, D.W. A qualidade da água na agricultura. 2 ed. Campina Grande: UFPB, 1999. 153p.

CFSEMG - Comissão de Fertilidade do Solo do Estado de Minas Gerais. Recomendações para o uso de corretivo e fertilizantes em Minas Gerais.- $5^{\text {a }}$ aproximação. Viçosa, Ed. universitária: 1999.359p

Cromer, R.N.; Tompkins, D.; Barr, N.J.; Hopmans, P. Irrigation of Monterey pine with wastewater: effect on soil chemistry and groundwater composition. Journal of Environmental Quality, Madison, v.13, n.4, p.539-542, 1984.

EMBRAPA - Empresa Brasileira de Pesquisa Agropecuária. Manual de métodos de análise do solo. 2.ed. Rio de Janeiro: EMBRAPA - Centro Nacional de Pesquisa de Solos, 1997. $247 \mathrm{p}$.

Falkiner, R.A.; Smith, C.J. Changes in soil chemistry in effluentirrigated Pinus radiata and Eucalyptus grandis. Australian Journal of Soil Research, Victoria, v.35, p.131-147, 1997.

Feigin, A.; Ravina, I.; Shalhevet, J. Irrigation with treated sewage effluent: management for environmental protection. Berlin: Springer-Verlag, 1991, 233p.

Fonseca, A.F. Disponibilidade de nitrogênio, alterações nas características químicas do solo e do milho pela aplicação de efluente de esgoto tratado. Piracicaba: ESALQ. 2001. 110p. Dissertação Mestrado

FUNARBE. SAEG - Sistema para análises estatísticas - versão 5.0. Viçosa: Fundação Arthur Bernardes. 1993. 80p.

Garcia, G.O. Alterações químicas, físicas e mobilidade de íons no solo decorrentes da aplicação de água residuária da lavagem e despolpa dos frutos do cafeeiro conilon. Viçosa: UFV. 2003. 100p. Dissertação Mestrado

Johns, G.G.; McConchie, D.M. Irrigation of bananas with secondary treated sewage effluent. I. Field evaluation of effect on plant nutrients and additional elements in leaf, pulp and soil. Australian Journal of Agricultural Research, Victoria, v.45, n. 7, p.1601-1617, 1994a.

Johns, G.G.; McConchie, D.M. Irrigation of bananas with secondary treated sewage effluent. II. Effect on plant nutrients, additional elements and pesticide residues in plants, soil and leachate using drainage lysimeters. Australian Journal of Agricultural Research, Victoria, v.45, p.1619-1638, 1994b. 
Latterell, J.J.; Dowdy, R.H.; Clapp, C.E.; Larson, W.E.; Linden, D.R. Distribution of phosphorus in soils irrigated with municipal waste-water effluent: a 5-year study. Journal of Environmental Quality, Madison, v.11, p.124-128, 1982.

Metcalf \& Eddy. Wastewater engineering: treatment, disposal, and reuse, New York: McGraw - Hill Inc., 1991. 1334p.

Pizarro, F. Riegos localizados de alta frecuencia. Madrid: Ediciones Mundi-Prensa, 2. ed., 1990.471p.

Rezende, A.A.P. Fertirrigação do eucalipto com efluente tratado de fábrica de celulose kraft branqueada. Viçosa: UFV. 2003, 152p. Tese Doutorado

Schipper, L.A.; Williamson, J.C.; Kettles, H.A; Speir, T.W. Impact of land applied tertiary-treated effluent on soil biochemical properties. Journal of Environmental Quality, Madison, v.25, n.5, p.1073-1077, 1996.

Smith, C.J.; Hopmans, P.; Cook, F.J. Accumulation of Cr, Pb, $\mathrm{Cu}, \mathrm{Ni}, \mathrm{Zn}$ and $\mathrm{Cd}$ in soil following irrigation with treated urban effluent in Australia. Environmental Pollution, v.94, n.3, p.317-323, 1996.
Speir, T.W.; van Schaik, A.P.; Kettles, H.A.; Vicent, K.W.; Campbell, D.J. Soil and stream-water impacts of sewage effluent irrigation onto steeply sloping land. Journal of Environmental Quality, Madison, v.28, n. 4, p.1105-1114, 1999.

Stewart, H.T.L.; Hopmans, P.; Flinn, D.W. Nutrient accumulation in trees and soil following irrigation with municipal effluent in Australia. Environmental Pollution, v.63, n.2, p.155-177, 1990.

van der Hoek, W.; Hassan, U. M.; Ensink, J. H. J.; Feenstra, S.; Raschid-Sally, L.; Munir, S.; Aslam, R.; Alim, N.; Hussain, R.; Matsuno, Y. Urban Wastewater: A valuable resource for agriculture; a case study from Horoonabad, Pakistan. Research Report 63. Colombo: International Water Management Institute. 2002

Vieira, E.O. Índices de lixiviação e modelagem do transporte de pesticidas no solo. Viçosa: UFV. 2003. 184p. Tese Doutorado 